\title{
ON MEAN ERGODIC CONVERGENCE IN THE CALKIN ALGEBRAS
}

\author{
MARCH T. BOEDIHARDJO AND WILLIAM B. JOHNSON
}

(Communicated by Thomas Schlumprecht)

\begin{abstract}
In this paper we give a geometric characterization of mean ergodic convergence in the Calkin algebras for Banach spaces that have the bounded compact approximation property.
\end{abstract}

\section{INTRODUCTION}

Let $X$ be a real or complex Banach space and let $B(X)$ be the algebra of all bounded linear operators on $X$. Suppose that $T \in B(X)$ and consider the sequence

$$
M_{n}(T):=\frac{I+T+\ldots+T^{n}}{n+1}, \quad n \geq 1 .
$$

In [3], Dunford considered the norm convergence of $\left(M_{n}(T)\right)_{n}$ and established the following characterizations.

Theorem 1.1. Suppose that $X$ is a complex Banach space and that $T \in B(X)$ satisfies $\frac{\left\|T^{n}\right\|}{n} \rightarrow 0$. Then the following conditions are equivalent.

(1) $\left(M_{n}(T)\right)_{n}$ converges in norm to an element in $B(X)$.

(2) 1 is a simple pole of the resolvent of $T$ or 1 is in the resolvent set of $T$.

(3) $(I-T)^{2}$ has closed range.

It was then discovered by Lin in [6] that $I-T$ having closed range is also an equivalent condition. Moreover, Lin's argument worked also for real Banach spaces. This result was later improved by Mbekhta and Zemánek in [9], in which they showed that $(I-T)^{m}$ having closed range, where $m \geq 1$, are also equivalent conditions. More precisely,

Theorem 1.2. Let $m \geq 1$. Suppose that $X$ is a real or complex Banach space and that $T \in B(X)$ satisfies $\frac{\left\|T^{n}\right\|}{n} \rightarrow 0$. Then the sequence $\left(M_{n}(T)\right)_{n}$ converges in norm to an element in $B(X)$ if and only if $(I-T)^{m}$ has closed range.

Let $K(X)$ be the closed ideal of compact operators in $B(X)$. If $T \in B(X)$, then its image in the Calkin algebra $B(X) / K(X)$ is denoted by $\dot{T}$. By Dunford's

\footnotetext{
Received by the editors March 18, 2013 and, in revised form, December 7, 2013 and December 18, 2013.

2010 Mathematics Subject Classification. Primary 46B08, 47A35, 47B07.

Key words and phrases. Mean ergodic convergence, Calkin algebra, essential maximality, essential norm, compact approximation property.

The first author was supported in part by the N. W. Naugle Fellowship and the A. G. \& M. E. Owen Chair in the Department of Mathematics, Texas A \& M University.

The second author was supported in part by NSF DMS-1301604.
} 
Theorem 1.1 or by an analogous version for Banach algebras (without condition (3)), when $X$ is a complex Banach space and $\frac{\left\|\dot{T}^{n}\right\|}{n} \rightarrow 0$, the convergence of $\left(M_{n}(\dot{T})\right)_{n}$ in the Calkin algebra is equivalent to 1 being a simple pole of the resolvent of $\dot{T}$ or being in the resolvent set of $\dot{T}$. But even if we are given that the limit $\dot{P} \in B(X) / K(X)$ of $\left(M_{n}(\dot{T})\right)_{n}$ exists, there is no obvious geometric interpretation of $\dot{P}$. In the context of Theorems 1.1 and 1.2, if the limit of $\left(M_{n}(T)\right)_{n}$ exists, then it is a projection onto $\operatorname{ker}(I-T)$. In the context of the Calkin algebra, the limit $\dot{P}$ is still an idempotent in $B(X) / K(X)$; hence by making a compact perturbation, we can assume that $P$ is an idempotent in $B(X)$ (see Lemma 2.6 below).

A natural question to ask is: what is the range of $P$ ? Although the range of $P$ is not unique (since $P$ is only unique up to a compact perturbation), it can be thought of as an analog of $\operatorname{ker}(I-T)$ in the Calkin algebra setting. If $T_{0} \in B(X)$, then $\operatorname{ker} T_{0}$ is the maximal subspace of $X$ on which $T_{0}=0$. This suggests that the analog of ker $T_{0}$ in the Calkin algebra setting is the maximal subspace of $X$ on which $T_{0}$ is compact. But the maximal subspace does not exist unless it is the whole space $X$. Thus, we introduce the following concept.

Let $X$ be a Banach space and let $(P)$ be a property that a subspace $M$ of $X$ may or may not have. We say that a subspace $M \subset X$ is an essentially maximal subspace of $X$ satisfying $(P)$ if it has $(P)$ and if every subspace $M_{0} \supset M$ having property $(P)$ satisfies $\operatorname{dim} M_{0} / M<\infty$.

Then the analog of ker $T_{0}$ in the Calkin algebra setting is an essentially maximal subspace of $X$ on which $T_{0}$ is compact. It turns that if such an analog for $I-T$ exists, then it is already sufficient for the convergence of $\left(M_{n}(\dot{T})\right)_{n}$ in the Calkin algebra (at least for a large class of Banach spaces), which is the main result of this paper.

Before stating this theorem, we recall that a Banach space $Z$ has the bounded compact approximation property (BCAP) if there is a uniformly bounded net $\left(S_{\alpha}\right)_{\alpha \in \Lambda}$ in $K(Z)$ converging strongly to the identity operator $I \in B(Z)$. It is always possible to choose $\Lambda$ to be the set of all finite dimensional subspaces of $Z$ directed by inclusion. If the net $\left(S_{\alpha}\right)_{\alpha \in \Lambda}$ can be chosen so that $\sup _{\alpha \in \Lambda}\left\|S_{\alpha}\right\| \leq \lambda$, then we say that $Z$ has the $\lambda$-BCAP. It is known that if a reflexive space has the BCAP, then the space has the 1-BCAP. For $T \in B(X)$, the essential norm $\|T\|_{e}$ is the norm of $\dot{T}$ in $B(X) / K(X)$.

Theorem 1.3. Let $m \geq 1$. Suppose that $X$ is a real or complex Banach space having the bounded compact approximation property. If $T \in B(X)$ satisfies $\frac{\left\|T^{n}\right\|_{e}}{n} \rightarrow$ 0 , then the following conditions are equivalent.

(1) The sequence $\left(M_{n}(\dot{T})\right)_{n}$ converges in norm to an element in $B(X) / K(X)$.

(2) There is an essentially maximal subspace of $X$ on which $(I-T)^{m}$ is compact.

The idea of the proof is to reduce Theorem 1.3 to Theorem 1.2 by constructing a Banach space $\widehat{X}$ and an embedding $f: B(X) / K(X) \rightarrow B(\widehat{X})$ so that if $T \in B(X)$ and there is an essentially maximal subspace $M$ of $X$ on which $T$ is compact, then $f(\dot{T})$ has closed range, and then applying Theorem 1.2 to $f(\dot{T})$. The BCAP of $X$ is used to show that $f$ is an embedding but is not used in the construction of $\widehat{X}$ and $f$. The construction of $f$ is based on the Calkin representation [1, Theorem 5.5]. 


\section{The Calkin representation for Banach spaces}

In this section, $X$ is a fixed infinite dimensional Banach space. Let $\Lambda_{0}$ be the set of all finite dimensional subspaces of $X$ directed by inclusion $\subset$. Then $\{\{\alpha \in$ $\left.\left.\Lambda_{0}: \alpha \supset \alpha_{0}\right\}: \alpha_{0} \in \Lambda_{0}\right\}$ is a filter base on $\Lambda_{0}$, so it is contained in an ultrafilter $U$ on $\Lambda_{0}$.

Let $Y$ be an arbitary infinite dimensional Banach space and let $\left(Y^{*}\right)^{U}$ be the ultrapower (see e.g. [2, Chapter 8]) of $Y^{*}$ with respect to $U$. (The ultrafilter $U$ and the directed set $\Lambda_{0}$ do not depend on $Y$.) If $\left(y_{\alpha}^{*}\right)_{\alpha \in \Lambda_{0}}$ is a bounded net in $Y^{*}$, then its image in $\left(Y^{*}\right)^{U}$ is denoted by $\left(y_{\alpha}^{*}\right)_{\alpha, U}$. Consider the (complemented) subspace

$$
\widehat{Y}:=\left\{\left(y_{\alpha}^{*}\right)_{\alpha, U} \in\left(Y^{*}\right)^{U}: w^{*}-\lim _{\alpha, U} y_{\alpha}^{*}=0\right\}
$$

of $\left(Y^{*}\right)^{U}$. Here $w^{*}-\lim _{\alpha, U} y_{\alpha}^{*}$ is the $w^{*}$-limit of $\left(y_{\alpha}^{*}\right)_{\alpha \in \Lambda_{0}}$ through $U$, which exists by the Banach-Alaoglu Theorem.

Whenever $T \in B(X, Y)$, we can define an operator $\widehat{T} \in B(\widehat{Y}, \widehat{X})$ by sending $\left(y_{\alpha}^{*}\right)_{\alpha, U}$ to $\left(T^{*} y_{\alpha}^{*}\right)_{\alpha, U}$. Note that if $K \in K(X, Y)$, then $\widehat{K}=0$, where $K(X, Y)$ denotes the space of all compact operators in $B(X, Y)$.

Theorem 2.1. Suppose that $X$ has the $\lambda-B C A P$. Then the operator

$$
f: B(X) / K(X) \rightarrow B(\widehat{X}), \dot{T} \mapsto \widehat{T},
$$

is a conjugate linear norm one $(\lambda+1)$-embedding into $B(\widehat{X})$ satisfying

$$
f(\dot{I})=I \text { and } f\left(\dot{T}_{1} \dot{T}_{2}\right)=f\left(\dot{T}_{2}\right) f\left(\dot{T}_{1}\right), \quad T_{1}, T_{2} \in B(X) .
$$

Proof. It is easy to verify that $f$ is a conjugate linear map, $f(\dot{I})=I$, and $f\left(\dot{T}_{1} \dot{T}_{2}\right)=$ $f\left(\dot{T}_{2}\right) f\left(\dot{T}_{1}\right)$ for $T_{1}, T_{2} \in B(X)$. If $T \in B(X)$, then clearly $\|f(\dot{T})\| \leq\|T\|$, and thus we also have $\|f(\dot{T})\| \leq\|T\|_{e}$. Hence $\|f\| \leq 1$. It remains to show that $f$ is a $(\lambda+1)$-embedding (i.e., $\left.\inf _{\|T\|_{e}>1}\|f(\dot{T})\| \geq(\lambda+1)^{-1}\right)$.

To do this, let $T \in B(X)$ satisfy $\|T\|_{e}>1$. Since $X$ has the $\lambda$-BCAP, we can find a net of operators $\left(S_{\alpha}\right)_{\alpha \in \Lambda_{0}} \subset K(X)$ converging strongly to $I$ such that $\sup \left\|S_{\alpha}\right\| \leq \lambda$. Then $\left\|T^{*}\left(I-S_{\alpha}\right)^{*}\right\|=\left\|\left(I-S_{\alpha}\right) T\right\| \geq\|T\|_{e}>1, \alpha \in \Lambda_{0}$. Thus, $\alpha \in \Lambda_{0}$

there exists $\left(x_{\alpha}^{*}\right)_{\alpha \in \Lambda_{0}} \subset X^{*}$ such that $\left\|x_{\alpha}^{*}\right\|=1$ and $\left\|T^{*}\left(I-S_{\alpha}\right)^{*} x_{\alpha}^{*}\right\|>1$ for $\alpha \in \Lambda_{0}$.

Note that for every $x \in X$,

$$
\limsup _{\alpha \in \Lambda_{0}}\left|\left\langle\left(I-S_{\alpha}\right)^{*} x_{\alpha}^{*}, x\right\rangle\right|=\limsup _{\alpha \in \Lambda_{0}}\left|\left\langle x_{\alpha}^{*},\left(I-S_{\alpha}\right) x\right\rangle\right| \leq \limsup _{\alpha \in \Lambda_{0}}\left\|\left(I-S_{\alpha}\right) x\right\|=0,
$$

and so the net $\left(\left(I-S_{\alpha}\right)^{*} x_{\alpha}^{*}\right)_{\alpha \in \Lambda_{0}}$ converges in the $w^{*}$-topology to 0 . By the construction of $U$, this implies that

$$
w^{*}-\lim _{\alpha, U}\left(I-S_{\alpha}\right)^{*} x_{\alpha}^{*}=0 .
$$

Therefore, due to the definition $f(\dot{T})=\widehat{T}$, we obtain

$$
\begin{aligned}
(1+\lambda)\|f(\dot{T})\| \geq\|f(\dot{T})\| \lim _{\alpha, U}\left\|\left(I-S_{\alpha}\right)^{*} x_{\alpha}^{*}\right\| & =\|f(\dot{T})\|\left\|\left(\left(I-S_{\alpha}\right)^{*} x_{\alpha}^{*}\right)_{\alpha, U}\right\| \\
& \geq\left\|f(\dot{T})\left(\left(I-S_{\alpha}\right)^{*} x_{\alpha}^{*}\right)_{\alpha, U}\right\| \\
& =\lim _{\alpha, U}\left\|T^{*}\left(I-S_{\alpha}\right)^{*} x_{\alpha}^{*}\right\| \geq 1 .
\end{aligned}
$$


It follows that $\|f(\dot{T})\| \geq(1+\lambda)^{-1}$ whenever $\|T\|_{e}>1$.

Remark 1. We do not know whether Theorem 2.1 is true without the hypothesis that $X$ has the BCAP.

Remark 2. The embedding in Theorem 2.1 is an isometry if the approximating net can be chosen so that $\left\|I-S_{\alpha}\right\|=1$ for every $\alpha$. This is the case if, for example, the space $X$ has a 1-unconditional basis. However, we do not know whether the embedding is an isometry if $X=L_{p}(0,1)$ with $p \neq 2$.

If $N$ is a subset of $Y^{*}$, then we can define a subset $N^{\prime}$ of $\widehat{Y}$ by

$$
N^{\prime}:=\left\{\left(y_{\alpha}^{*}\right)_{\alpha, U} \in \widehat{Y}: \lim _{\alpha, U} d\left(y_{\alpha}^{*}, N\right)=0\right\},
$$

where

$$
d\left(y_{\alpha}^{*}, N\right):=\inf _{z^{*} \in N}\left\|y_{\alpha}^{*}-z^{*}\right\| .
$$

Lemma 2.2. If $N$ is a $w^{*}$-closed subspace of $Y^{*}$, then for every $\left(y_{\alpha}^{*}\right)_{\alpha, U} \in \widehat{Y}$,

$$
d\left(\left(y_{\alpha}^{*}\right)_{\alpha, U}, N^{\prime}\right) \leq 2 \lim _{\alpha, U} d\left(y_{\alpha}^{*}, N\right) .
$$

Proof. Let $a=\lim _{\alpha, U} d\left(y_{\alpha}^{*}, N\right)$. Let $\delta>0$. Then

$$
A:=\left\{\alpha \in \Lambda: d\left(y_{\alpha}^{*}, N\right)<a+\delta\right\} \in U .
$$

Whenever $\alpha \in A,\left\|y_{\alpha}^{*}-z_{\alpha}^{*}\right\|<a+\delta$ for some $z_{\alpha}^{*} \in N$. If we take $z_{\alpha}^{*}=0$ for $\alpha \notin A$, then, since $\sup _{\alpha \in \Lambda}\left\|y_{\alpha}^{*}\right\|<\infty$,

$$
\sup _{\alpha \in \Lambda}\left\|z_{\alpha}^{*}\right\|=\sup _{\alpha \in A}\left\|z_{\alpha}^{*}\right\| \leq(a+\delta)+\sup _{\alpha \in A}\left\|y_{\alpha}^{*}\right\|<\infty .
$$

As a consequence, $\left(z_{\alpha}^{*}-w^{*}-\lim _{\beta, U} z_{\beta}^{*}\right)_{\alpha, U} \in N^{\prime}$, since $N$ is $w^{*}$-closed. Therefore,

$$
\begin{aligned}
d\left(\left(y_{\alpha}^{*}\right)_{\alpha, U}, N^{\prime}\right) & \leq d\left(\left(y_{\alpha}^{*}\right)_{\alpha, U},\left(z_{\alpha}^{*}-w^{*}-\lim _{\beta, U} z_{\beta}^{*}\right)_{\alpha, U}\right) \\
& =\lim _{\alpha, U}\left\|y_{\alpha}^{*}-z_{\alpha}^{*}+w^{*}-\lim _{\beta, U} z_{\beta}^{*}\right\| \\
& \leq \lim _{\alpha, U}\left\|y_{\alpha}^{*}-z_{\alpha}^{*}\right\|+\left\|w^{*}-\lim _{\beta, U} z_{\beta}^{*}\right\| \\
& \leq(a+\delta)+\left\|w^{*}-\lim _{\beta, U}\left(z_{\beta}^{*}-y_{\beta}^{*}\right)\right\| \\
& \leq(a+\delta)+\lim _{\beta, U}\left\|z_{\beta}^{*}-y_{\beta}^{*}\right\| \leq 2(a+\delta) .
\end{aligned}
$$

But $\delta$ can be arbitarily close to 0 , so $d\left(\left(y_{\alpha}^{*}\right)_{\alpha, U}, N^{\prime}\right) \leq 2 a=2 \lim _{\alpha, U} d\left(y_{\alpha}^{*}, N\right)$.

Proposition 2.3. If $X$ and $Y$ are infinite dimensional Banach spaces and if $T \in$ $B(X, Y)$ has closed range, then $\widehat{T} \in B(\widehat{Y}, \widehat{X})$ also has closed range. 
Proof. The operator $T$ has closed range, so $T^{*}$ also has closed range. Let $c=$ $\inf \left\{\left\|T^{*} y^{*}\right\|: y^{*} \in Y^{*}, d\left(y^{*}, \operatorname{ker} T^{*}\right)=1\right\}>0$. Then by Lemma 2.2, for every $\left(y_{\alpha}^{*}\right)_{\alpha, U} \in \widehat{Y}$,

$$
\left\|\widehat{T}\left(y_{\alpha}^{*}\right)_{\alpha, U}\right\|=\lim _{\alpha, U}\left\|T^{*} y_{\alpha}^{*}\right\| \geq c \lim _{\alpha, U} d\left(y_{\alpha}^{*}, \operatorname{ker} T^{*}\right) \geq \frac{c}{2} d\left(\left(y_{\alpha}^{*}\right)_{\alpha, U},\left(\operatorname{ker} T^{*}\right)^{\prime}\right) .
$$

But obviously $\left(\operatorname{ker} T^{*}\right)^{\prime} \subset \operatorname{ker} \widehat{T}$, and so

$$
\left\|\widehat{T}\left(y_{\alpha}^{*}\right)_{\alpha, U}\right\| \geq \frac{c}{2} d\left(\left(y_{\alpha}^{*}\right)_{\alpha, U}, \text { ker } \widehat{T}\right), \quad\left(y_{\alpha}^{*}\right)_{\alpha, U} \in \widehat{Y} .
$$

Hence $\widehat{T}$ has closed range.

Lemma 2.4. Suppose that $X \subset Y$ and that $T \in B(X)$. Let $T_{0} \in B(X, Y), x \mapsto T x$. Then $\widehat{T}_{0} \widehat{Y}=\widehat{T} \widehat{X}$.

Proof. If $\left(y_{\alpha}^{*}\right)_{\alpha, U} \in \widehat{Y}$, then for each $\alpha \in \Lambda$, we have $T_{0}^{*} y_{\alpha}^{*}=T^{*}\left(y_{\alpha \mid X}^{*}\right)$ and $\left(y_{\alpha \mid X}^{*}\right)_{\alpha, U} \in \widehat{X}$. Thus $\widehat{T}_{0}\left(y_{\alpha}^{*}\right)_{\alpha, U}=\left(T_{0}^{*} y_{\alpha}^{*}\right)_{\alpha, U}=\left(T^{*}\left(y_{\alpha \mid X}^{*}\right)\right)_{\alpha, U}=\widehat{T}\left(y_{\alpha \mid X}^{*}\right)_{\alpha, U} \in$ $\widehat{T} \widehat{X}$. Hence $\widehat{T_{0}} \widehat{Y} \subset \widehat{T} \widehat{X}$.

Conversely, if $\left(x_{\alpha}^{*}\right)_{\alpha, U} \in \widehat{X}$, then we can extend each $x_{\alpha}^{*}$ to an element $y_{\alpha}^{*} \in Y^{*}$ such that $\left\|y_{\alpha}^{*}\right\|=\left\|x_{\alpha}^{*}\right\|$. Thus we have $\left(y_{\alpha}^{*}-w^{*}-\lim _{\beta, U} y_{\beta}^{*}\right)_{\alpha, U} \in \widehat{Y}$. Note that

$$
T_{0}^{*}\left(w^{*}-\lim _{\beta, U} y_{\beta}^{*}\right)=w^{*}-\lim _{\beta, U} T_{0}^{*} y_{\beta}^{*}=w^{*}-\lim _{\beta, U} T^{*} x_{\beta}^{*}=T^{*}\left(w^{*}-\lim _{\beta, U} x_{\beta}^{*}\right)=0 .
$$

This implies that

$$
\begin{aligned}
\widehat{T}\left(x_{\alpha}^{*}\right)_{\alpha, U}=\left(T^{*} x_{\alpha}^{*}\right)_{\alpha, U} & =\left(T_{0}^{*} y_{\alpha}^{*}\right)_{\alpha, U} \\
& =\left(T_{0}^{*}\left(y_{\alpha}^{*}-w^{*}-\lim _{\beta, U} y_{\beta}^{*}\right)\right)_{\alpha, U} \\
& =\widehat{T}_{0}\left(y_{\alpha}^{*}-w^{*}-\lim _{\beta, U} y_{\beta}^{*}\right)_{\alpha, U} \in \widehat{T}_{0} \widehat{Y} .
\end{aligned}
$$

Therefore $\widehat{T} \widehat{X} \subset \widehat{T}_{0} \widehat{Y}$.

Proposition 2.5. Suppose that $T \in B(X)$ and that there exists an essentially maximal subspace $M$ of $X$ on which $T$ is compact. Then $\widehat{T}$ has closed range.

Proof. Without loss of generality, we may assume that $X$ is a subspace of $Y=$ $\ell_{\infty}(J)$ for some set $J$. Define $T_{0} \in B\left(X, \ell_{\infty}(J)\right), x \mapsto T x$. Then by assumption, there is an essentially maximal subspace $M$ of $X$ on which $T_{0}$ is compact. By [7. Theorem 3.3], there exists $K \in K\left(X, \ell_{\infty}(J)\right)$ such that $K_{\mid M}=T_{0 \mid M}$.

We now show that $T_{0}-K \in B\left(X, \ell_{\infty}(J)\right)$ has closed range. Since $M \subset$ $\operatorname{ker}\left(T_{0}-K\right)$ and $M$ is an essentially maximal subspace of $X$ on which $T_{0}-K$ is compact, $\operatorname{ker}\left(T_{0}-K\right)$ is an essentially maximal subspace of $X$ on which $T_{0}-K$ is compact.

Let $\pi$ be the quotient map from $X$ onto $X / \operatorname{ker}\left(T_{0}-K\right)$. Define the (one-toone) operator $R: X / \operatorname{ker}\left(T_{0}-K\right) \rightarrow \ell_{\infty}(J), \pi x \mapsto\left(T_{0}-K\right) x$. If $R$ does not have closed range, then by [8, Proposition 2.c.4], $R$ is compact on an infinite dimensional subspace $V$ of $X / \operatorname{ker}\left(T_{0}-K\right)$. Hence, $T_{0}-K$ is compact on $\pi^{-1} V$ and so by the essential maximality of $\operatorname{ker}\left(T_{0}-K\right)$, we have $\operatorname{dim} \pi^{-1} V / \operatorname{ker}\left(T_{0}-K\right)<\infty$. Thus, $V=\pi^{-1} V / \operatorname{ker}\left(T_{0}-K\right)$ is finite dimensional, which contradicts the definition of $V$. 
Therefore, $R$ has closed range and so $T_{0}-K$ also has closed range. By Proposition 2.3. $\widehat{T_{0}-K}$ has closed range. But $\widehat{K}=0$, so $\widehat{T}_{0}$ has closed range and by Lemma 2.4, $\widehat{T}$ has closed range.

Lemma 2.6. Suppose that $P \in B(X)$ and that $\dot{P}$ is an idempotent in $B(X) / K(X)$. Then $P$ is the sum of an idempotent in $B(X)$ and a compact operator on $X$.

Proof. We first treat the case where the scalar field is $\mathbb{C}$. From Fredholm theory (see e.g. [5, Chapters XI and XVII]), we know that since $\sigma(\dot{P}) \subset\{0,1\}$, the only possible cluster points of $\sigma(P)$ are 0 and 1 . Thus, there exists $0<r<1$ such that $\{z \in \mathbb{C}:|z-1|=r\} \cap \sigma(P)=\emptyset$. Then $\dot{P}=\frac{1}{2 \pi i} \oint_{|z-1|=r}(z \dot{I}-\dot{P})^{-1} d z$ and so $P-\frac{1}{2 \pi i} \oint_{|z-1|=r}(z I-P)^{-1} d z \in K(X)$. But $\frac{1}{2 \pi i} \oint_{|z-1|=r}(z I-P)^{-1} d z$ is an idempotent in $B(X)$ (see e.g. [10, Theorem 2.7]). This completes the proof in the complex case.

If $X$ is a real Banach space, then let $X_{C}$ and $P_{C}$ be the complexifications (see 4 , page 266]) of $X$ and $P$, respectively. Thus, $\dot{P}_{C}$ is an idempotent in $B\left(X_{C}\right) / K\left(X_{C}\right)$. Since the only possible cluster points of $\sigma\left(P_{C}\right)$ are 0 and 1 , there exists a closed rectangle $R$ in the complex plane symmetric with respect to the real axis such that 1 is in the interior of $R, 0$ is in the exterior of $R$, and $\sigma\left(P_{C}\right)$ is disjoint from the boundary $\partial R$ of $R$. By [4, Lemma 3.4], the idempotent $\frac{1}{2 \pi i} \oint_{\partial R}\left(z I-P_{C}\right)^{-1} d z$ in $B\left(X_{C}\right)$ is induced by an idempotent $P_{0}$ in $B(X)$. Since $P_{C}-\frac{1}{2 \pi i} \oint_{\partial R}\left(z I-P_{C}\right)^{-1} d z \in$ $K\left(X_{C}\right)$, we see that $P-P_{0} \in K(X)$.

Proof of Theorem 1.3. "(1) $\Rightarrow(2) "$ : Let $\dot{P}:=\lim _{n \rightarrow \infty} \frac{\dot{I}+\dot{T}+\ldots+\dot{T}^{n}}{n+1}$.

Since $\lim _{n \rightarrow \infty} \frac{\left\|\dot{T}^{n}\right\|}{n}=0$,

$$
(\dot{I}-\dot{T}) \dot{P}=\lim _{n \rightarrow \infty}(\dot{I}-\dot{T}) \frac{\dot{I}+\dot{T}+\ldots+\dot{T}^{n}}{n+1}=\lim _{n \rightarrow \infty} \frac{\dot{I}-\dot{T}^{n+1}}{n+1}=0 .
$$

Thus $\dot{T} \dot{P}=\dot{P}$, and so

$$
\dot{P}^{2}=\lim _{n \rightarrow \infty} \frac{\dot{P}+\dot{T} \dot{P}+\ldots+\dot{T}^{n} \dot{P}}{n+1}=\lim _{n \rightarrow \infty} \frac{(n+1) \dot{P}}{n+1}=\dot{P} .
$$

Hence $\dot{P}$ is an idempotent in $B(X) / K(X)$. By Lemma 2.6, there exists an idempotent $P_{0}$ in $B(X)$ such that $P-P_{0} \in K(X)$. Replacing $P$ with $P_{0}$, we can assume without loss of generality that $P$ is an idempotent in $B(X)$. Equation (2.1) also implies that $(I-T) P \in K(X)$, which means that $I-T$ is compact on $P X$. Hence $(I-T)^{m}$ is compact on $P X$.

We now show that $P X$ is an essentially maximal subspace of $X$ on which $(I-T)^{m}$ is compact. Suppose that $(I-T)^{m}$ is compact on a subspace $M_{0}$ of $X$ containing $P X$. Let

$$
f_{n}(z):=\frac{n+(n-1) z+(n-2) z^{2}+\ldots+z^{n-1}}{n+1}, \quad z \in \mathbb{C}, n \geq 1 .
$$

Note that $\dot{I}-\frac{\dot{I}+\dot{T}+\ldots+\dot{T}^{n}}{n+1}=(\dot{I}-\dot{T}) f_{n}(\dot{T})$. Therefore,

$$
\dot{I}-\dot{P}=(\dot{I}-\dot{P})^{m}=\lim _{n \rightarrow \infty} f_{n}(\dot{T})^{m}(\dot{I}-\dot{T})^{m},
$$


and so

$$
\lim _{n \rightarrow \infty}\left\|(I-P)-\left(f_{n}(T)^{m}(I-T)^{m}+K_{n}\right)\right\|=0,
$$

for some $K_{1}, K_{2}, \ldots \in K(X)$.

Since $(I-T)^{m}$ is compact on $M_{0}$, the operator $f_{n}(T)^{m}(I-T)^{m}$ is compact on $M_{0}$ and so is $f_{n}(T)^{m}(I-T)^{m}+K_{n}$ on $M_{0}$. Thus $(I-P)_{\mid M_{0}}$ is the norm limit of a sequence in $K\left(M_{0}, X\right)$, and so $I-P$ is compact on $M_{0}$. Since $P X \subset M_{0}$, we have that $(I-P) M_{0} \subset M_{0}$. Therefore, $(I-P)_{\mid(I-P) M_{0}}=I_{\mid(I-P) M_{0}}$ is compact, and so $(I-P) M_{0}$ is finite dimensional. In other words, $\operatorname{dim} M_{0} / P X<\infty$.

" $(2) \Rightarrow(1) "$ : By Proposition 2.5. $\left(\widehat{I-T)^{m}}=(I-\widehat{T})^{m}\right.$ has closed range. Since by assumption $\lim _{n \rightarrow \infty} \frac{\left\|T^{n}\right\|_{e}}{n}=0, \lim _{n \rightarrow \infty} \frac{\left\|\widehat{T}^{n}\right\|}{n}=\lim _{n \rightarrow \infty} \frac{\left\|\widehat{T}^{n}\right\|}{n}=0$. By MbekhtaZemánek's Theorem [1.2, the sequence $\left(M_{n}(\widehat{T})\right)_{n}$ converges in norm to an element in $B(\widehat{X})$. By Theorem 2.1 the result follows.

\section{ACKNOWLEDGMENT}

The authors thank C. Foias and C. Pearcy for helpful discussions.

\section{REFERENCES}

[1] J. W. Calkin, Two-sided ideals and congruences in the ring of bounded operators in Hilbert space, Ann. of Math. (2) 42 (1941), 839-873. MR0005790(3,208c)

[2] Joe Diestel, Hans Jarchow, and Andrew Tonge, Absolutely summing operators, Cambridge Studies in Advanced Mathematics, vol. 43, Cambridge University Press, Cambridge, 1995. MR.1342297 (96i:46001)

[3] Nelson Dunford, Spectral theory. I. Convergence to projections, Trans. Amer. Math. Soc. 54 (1943), 185-217. MR0008642 (5,39c)

[4] I. S. Ėdel'štĕn and P. Wojtaszczyk, On projections and unconditional bases in direct sums of Banach spaces, Studia Math. 56 (1976), no. 3, 263-276. MR0425585 (54 \#13539)

[5] Israel Gohberg, Seymour Goldberg, and Marinus A. Kaashoek, Classes of linear operators. Vol. I, Operator Theory: Advances and Applications, vol. 49, Birkhäuser Verlag, Basel, 1990. MR.1130394 (93d:47002)

[6] Michael Lin, On the uniform ergodic theorem, Proc. Amer. Math. Soc. 43 (1974), 337-340. MR0417821(54 \#5869)

[7] Joram Lindenstrauss, Extension of compact operators, Mem. Amer. Math. Soc. No. 48 (1964), 112 pp. MR0179580 (31 \#3828)

[8] Joram Lindenstrauss and Lior Tzafriri, Classical Banach spaces. I, Sequence spaces, Ergebnisse der Mathematik und ihrer Grenzgebiete, Vol. 92, Springer-Verlag, Berlin, 1977. MR.0500056 (58 \#17766)

[9] Mostafa Mbekhta and Jaroslav Zemánek, Sur le théorème ergodique uniforme et le spectre (French, with English and French summaries), C. R. Acad. Sci. Paris Sér. I Math. 317 (1993), no. 12, 1155-1158. MR1257230 (95b:47010)

[10] Heydar Radjavi and Peter Rosenthal, Invariant subspaces, 2nd ed., Dover Publications Inc., Mineola, NY, 2003. MR2003221 (2004e:47010)

Department of Mathematics, Texas A\&M University, College Station, Texas 77843

E-mail address: march@math.tamu.edu

Department of Mathematics, Texas A\&M University, College Station, Texas 77843

E-mail address: johnson@math.tamu.edu 